\title{
Biologia do Triatoma nitida Usinger, 1939 em Laboratório (Hemiptera: Reduviidae)
}

\author{
Cleber Galvão, José Jurberg, Vanda Cunha, Rubens Pinto de Mello*
}

Laboratório Nacional e Internacional de Referência em Taxonomia de Triatomíneos *Laboratório de Díptera, Departamento de Entomologia, Instituto Oswaldo Cruz, Av. Brasil 4365, 21045-900 Rio de Janeiro, RJ, Brasil

Biology of Triatoma nitida Usinger, 1939 under Laboratory Conditions -Triatoma nitida is a wild species occurring in Mexico and Central America. In order to establish the length of its life cycle and transmission potential, the following parameters were observed: the incubation period, the interval between hatching, or moulting, and the first feeding; the number of blood meals and the time of development. The time-lapse before the bite, the length of feeding and the interval between the end of the blood meal and defecation, as well as the site of defecation were also analyzed. Average length of the egg incubation period was 18.2 days. Time interval between the food source offering and the bite was less than 4 min in $60 \%$ of the analyzed cases, except in the fifth instar, where only $38 \%$ of the insects began feeding in less than 5 min. The blood-sucking period was long and rising until the fifth instar, decreasing in adults, and ranging from $1 \mathrm{~min}$ to 2 and a half hours. Only 26\% of the blood meals were followed by defecations within 20 min. The average length of the life cycle was 897.5 days.

Key words: life cycle - Triatoma nitida - Triatominae - Chagas' disease

Triatoma nitida Usinger, 1939 é uma espécie silvestre, de ocorrência assinalada na Costa Rica, Guatemala, Honduras e México. Dias (1952) publicou uma lista da distribuição das espécies centroamericanas e assinalou as localidades onde foi verificada a infecção de triatomíneos pelo Trypanosoma cruzi. T. nitida foi encontrado infectado pelo flagelado na Guatemala, porém em Honduras nenhum dos espécimes examinados apresentou infecção. Leon (1959) chamou atenção para o alto grau de infecção dessa espécie na Guatemala, onde $83 \%$ dos espécimes capturados encontravamse positivos para T. cruzi. Gonzalez-Angulo e Ryckman (1967) encontraram, pela primeira vez no México (Yucatan), representantes dessa espécie.

Sob o ponto de vista morfológico e taxonômico, esse triatomíneo foi analisado nos trabalhos de Lent e Wygodzinsky (1979), Jurberg et al. (1991), Lent e Jurberg (1992), entretanto sua biologia, hospedeiros naturais e habitats são pouco conhecidos.

Com intuito de ampliar os parâmetros que permitem a definição precisa dessa espécie, registrou-se o período de desenvolvimento e avaliou-se o potencial vetorial em condições de laboratório. Dentro deste propósito, observaram-se: o período de incubação, o intervalo entre a eclosão ou muda

Com auxílio do CNPq, FAPERJ e convênio BIRD/FNS/ FIOCRUZ 027/93 - Parte da tese de Mestrado do $1^{\circ}$ autor Recebido em 26 de janeiro de 1995

Aceito em 10 de maio de 1995 e o primeiro repasto; o número de repastos e 0 período de desenvolvimento de ovo a adulto em 92 espécimes. A observação de 1856 repastos sanguíneos possibilitou o registro do intervalo entre o oferecimento da fonte alimentar e a picada; o tempo de duração do repasto; o intervalo decorrido entre o fim do repasto e a defecação; e o local da defecação.

\section{MATERIAIS E MÉTODOS}

Os insetos estudados eram provenientes de uma colônia aclimatada no insetário do Laboratório Nacional e Internacional de Referência em Taxonomia de Triatomíneos, do Departamento de Entomologia do IOC, onde são mantidos em temperatura ambiente com alimentação quinzenal em pombos (Columba livia).

Quinze espécimes de $5^{\circ}$ estádio foram retirados da colônia e mantidos em um cristalizador de vidro transparente, de $20 \mathrm{~cm}$ de altura por $17 \mathrm{~cm}$ de diâmetro, e observados diariamente até a ocorrência da muda imaginal. Destes, cinco fêmeas e três machos foram transferidos para um recipiente metálico de $21 \mathrm{~cm}$ de altura por $16 \mathrm{~cm}$ de diâmetro, com o fundo de tela de arame, visando facilitar a coleta dos ovos.

Os ovos coletados diariamente, eram agrupados de acordo com a data da postura e aclimatados em estufa B.O.D. a temperatura de $28 \pm 1^{\circ} \mathrm{C}$ e 80 $\pm 5 \%$ de U.R., com fotoperíodo controlado, alternando-se 12 h de luz com 12 de obscuridade. 
Dos 135 ovos separados, 92 eclodiram. As ninfas recém emergidas foram individualizadas em frascos de Borrel $(9 \mathrm{~cm}$ de altura $\times 3 \mathrm{~cm}$ de diâmetro) numerados, forrados no fundo por papel de filtro e com uma tira do mesmo papel para absorver o excesso de umidade e proporcionar maior área de deslocamento aos insetos. Os frascos foram fechados com telas de náilon fixadas por elásticos. A alimentação foi oferecida diariamente até a ocorrência do primeiro repasto de cada fase, passando então a ser semanal. Foi realizada em camundongos albinos (Mus musculus), que tinham as patas imobilizadas por meio de fita adesiva. No dorso do animal afixava-se uma tira de papel para servir de apoio ao inseto durante o repasto. O tempo de oferecimento da fonte alimentar foi de $20 \mathrm{~min}$., sendo o tempo de sucção rigorosamente cronometrado, desde o momento da picada; após o término do repasto, aguardavam-se $20 \mathrm{~min}$. para verificar o local (sobre a fonte ou não) e o tempo decorrido até o momento da defecação.

\section{RESULTADOS E DISCUSSÃO}

Ovos - O periodo de incubação dos 92 ovos observados variou de 13 a 21 dias, com média de $18.2\left(\mathrm{~S}=1.49 \mathrm{e} \mathrm{S}^{2}=2.24\right)$. Resultados semelhantes foram obtidos por Carcavallo e Martinez (1972) para T. delpontei Romaña \& Abalos, 1947; por Rabinovich (1972) para T. infestans (Klug, 1834) e por Silva e Silva (1991) para $T$. dimidiata (Latreille, 1811) criados em condições similares.

Realização do primeiro repasto - Através do oferecimento diário da fonte alimentar foi possível registrar o intervalo decorrido entre a eclosão, ou muda. c o primeiro repasto. Os resultados obtidos mostraram que o intervalo foi crescente, apesar de a média do $2^{\circ}$ estádio ter sido menor que a do $1^{\circ}$. Todas as ninfas precisaram de ao menos um dia de "repouso" pré-alimentar após a eclosão, ou muda, e os adultos de pelo menos dois dias (Tabela I). Lafetá (1966) demonstrou que, à temperatura de $25^{\circ} \mathrm{C}$ e $80 \%$ de U.R, as ninfas de $1^{\circ}$ estádio de Panstrongylus megistus (Burmeister, 1835) só se alimentaram a partir do quarto dia de vida, e que no sexto dia a alimentação foi realizada por $80 \%$ dos espécimes. Segundo Perlowagora-Szumlewicz (1969). a primeira alimentação do T. infestans teria importância vital para o surgimento precoce dos adultos. e que o quarto dia após a eclosão seria o ideal para promover esta possibilidade. Leopoldoe-Silva et al. (1990) testaram esta hipótese em ninfas de T. infestans e Rhodnius prolixus Stal, 1859 e demonstraram que não houve diferença significativa nos resultados obtidos para os insetos alimentados no $4^{\circ}$, no $8^{\circ}$ e no $12^{\circ}$ dia.

Tempo decorrido entre o oferecimento da fonte alimentar e a picada - $O$ registro do tempo decorrido entre o oferecimento da fonte c a picada

\section{TABELA I}

Intervalo de tempo (em dias) decorrido entre a eclosão, ou muda de Triatoma nitida, e o primeiro repasto

\begin{tabular}{|c|c|c|c|c|c|c|}
\hline Fases & Min. & Máx. & $\overline{\mathrm{X}}$ & $\mathrm{S}$ & $s^{2}$ & \\
\hline $1^{0}$ estádio & 1 & 22 & 5,6 & 3,36 & 11,34 & 1 \\
\hline $2^{\circ}$ estádio & 1 & & 3,7 & 2,45 & 6 & 72 \\
\hline $3^{\circ}$ estádio & 1 & 29 & 5,7 & 4,46 & 19,92 & 68 \\
\hline $4^{\circ}$ estádio & 1 & 26 & 8,3 & 5,56 & 30,97 & 60 \\
\hline $5^{\circ}$ estádio & 1 & 50 & 10,8 & 9,08 & 82,58 & 56 \\
\hline$\Delta$ dultos & 2 & 55 & 14,1 & 10,1 & 102,17 & 35 \\
\hline
\end{tabular}

permitiu avaliar a agressividade e voracidade na procura pelo alimento. T. nitida demonstrou pouca agressividade, apesar disso, em cerca de $60 \%$ dos casos observados, o intervalo entre o oferecimento e a picada foi inferior a 4,5 min.; e em apenas $7 \%$ este intervalo foi superior a $10 \mathrm{~min}$. Este foi o padrão para todas as fases, exceto para o $5^{\circ}$ estádio, onde apenas $38 \%$ aceitaram a alimentação em menos de 5 min. e 16\% ultrapassaram os $10 \mathrm{~min}$. (Fig. 1). Deve-se considerar que a variabilidade no período de tempo que antecedeu a picada, ccrtamente sofreu a influência do manuseio e da mudança de temperatura e umidade ocasionadas pela retirada dos insetos da estufa para possibilitar as observações.

Tempo de suç̧ão e eliminação das dejeções A realização do repasto sanguíneo possibilita o contato entre o vetor e o hospedeiro; a conseqüente defecação é o meio mais comum de exposição do hospedeiro ao T. cruzi. Estudos sobre o tempo de alimentação e tempo de defecação são de grande importância para ampliar os conhecimentos sobre a transmissão do T. cruzi e apontar as espécies mais capacitadas a desempenhar com eficiência seu potencial vetorial. Já em 1949 Dias e Chandler alertavam para a importância dos hábitos de defecação dos triatomíneos, porém, foi Wood (1951) o primeiro a desenvolver experimentos sobre tais hábitos.

A duração do repasto foi crescente do $1^{\circ}$ até o $5^{\circ}$ estádio; na fase adulta sofreu pequena redução e ficou mais próxima dos resultados obtidos para o $4^{\circ}$ estádio (Fig. 2). Esta redução no tempo de sucção dos adultos está diretamente relacionada à quantidade de sangue ingerida. Esse aspecto já foi demonstrado por Pippin (1970), em T. sanguisuga Usinger, 1944, T. gerstaeckeri (Stal, 1859) e $R$. prolixus; por Wood (1976), em T. protracta (Uhler, 1894); por Zeledón et al. (1977) em $R$. prolixus, T. infestans e T. dimidiata; por Zárate et al. (1984) para T. barberi Usinger, 1939 e mais recentemente por Rocha et al. (1994) para R. pictipes Stal, 1872 . 


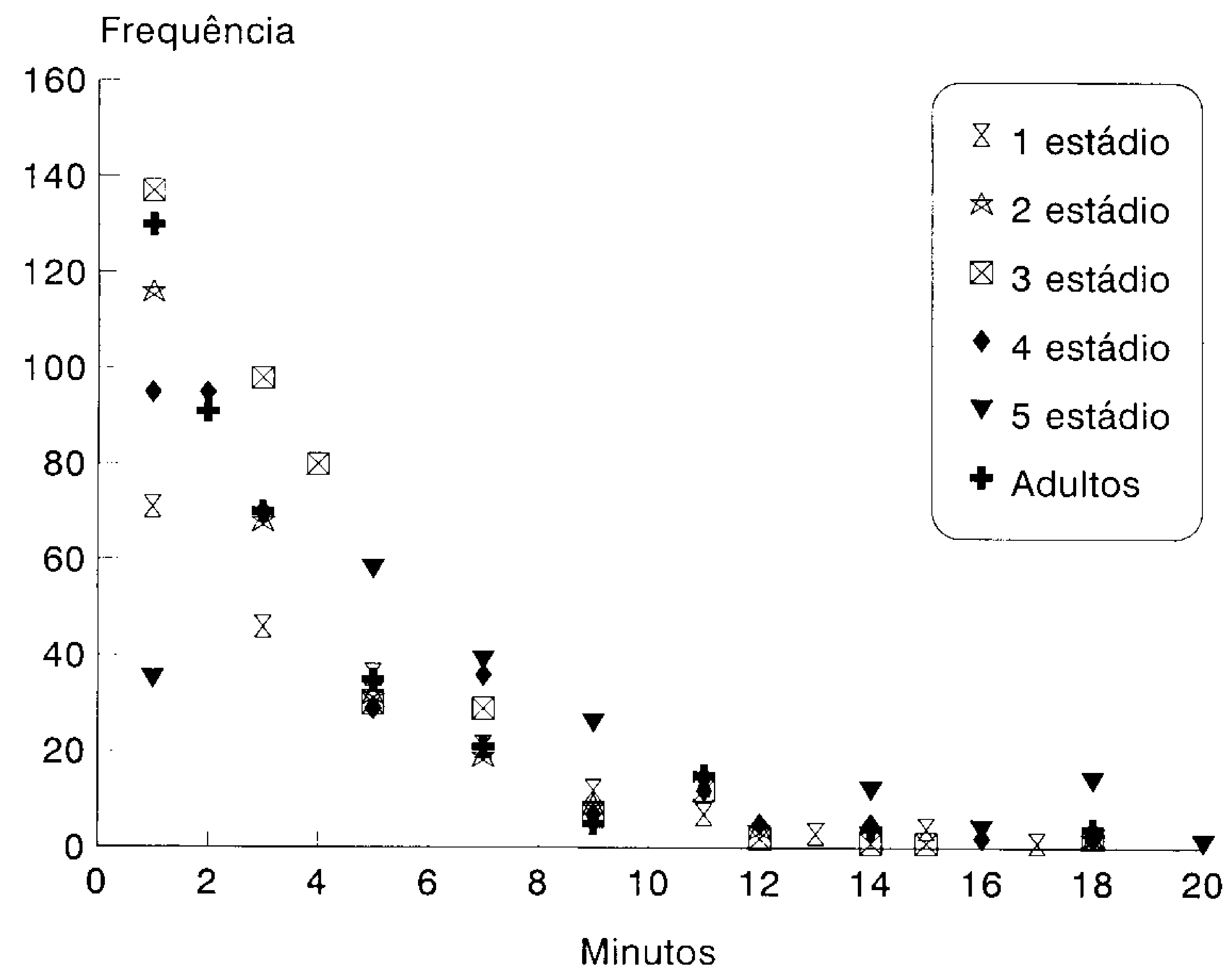

Fig. 1: tempo decorrido entre o oferecimento da fonte alimentar e a picada em todas as fases do desenvolvimento de Triatoma nitida.

Brasileiro e Perondini (1976), observando o tempo de sucção e repleção de ninfas de $4^{\circ}$ e $5^{\circ}$ estádios de T. brasiliensis Neiva, 1911, notaram que "até os $30 \mathrm{~min}$. de sucção o aumento da quantidade ingerida é aproximadamente linear. A partir dai tende-se a uma estabilização". Estabelecendo uma relação entre peso corporal e quantidade de sangue ingerida, esses autores, verificaram que as ninfas de $4^{\circ}$ estádio foram capazes de sugar quantidade de sangue 4,6 vezes superior ao próprio peso, enquanto que para o $5^{\circ}$ estádio esta quantidade foi de 3,5 vezes. Costa e Jurberg (1990) estabeleceram esta relação para todas as fases do desenvolvimento de Cavernicola lenti Barrett \& Arias, 1985 e observaram que o $3^{\circ}$ estádio apresentou a maior capacidade de ingestão proporcional de sangue.

O tempo de defecação é crucial na transmissão do T. cruzi, sendo mais eficazes as espécies que defecam durante ou logo após o repasto. Esse aspecto foi discutido, entre outros, por Dias (1956), Zeledón et al. (1977), Ozete (1981), Zárate et al. (1984), Kirk e Schofield (1987), Costa e Jurberg (1990) e Rocha et al. (1994).
Neste experimento, dos 1856 repastos realizados, $484(26 \%)$ foram seguidos de defecações no prazo de 20 min., e destas, apenas 152 (31.4\%) foram depositadas diretamente sobre a fonte. Os resultados obtidos para cada fase do ciclo encontram-se na Fig. 3 e indicam que, sob este aspecto, T. nitida apresenta baixo potencial vetorial.

Número de repastos realizados em cada fase do ciclo de vida - $\mathrm{O}$ oferecimento semanal da alimentação e a manutenção dos espécimes que se recusavam a sugar resultaram em grandes variações individuais no número de repastos realizados, porém, deste modo, as características individuais foram mantidas, evitando-se a manipulação dos resultados. A Tabela II mostra os resultados obtidos para este parâmetro em cada fase do desenvolvimento. Segundo Juarez (1970), a avaliação do número de repastos tem grande importância epidemiológica, uma vez que quanto mais contatos ocorrerem entre vetor e hospedeiro, maior será a possibilidade de infecção ou transmissão do T. cruzi. Em T. nitida, o grande número de repastos realizados aumentaria a probabilidade de infecção e/ou transmissão, porém na natureza aumen- 
Frequência

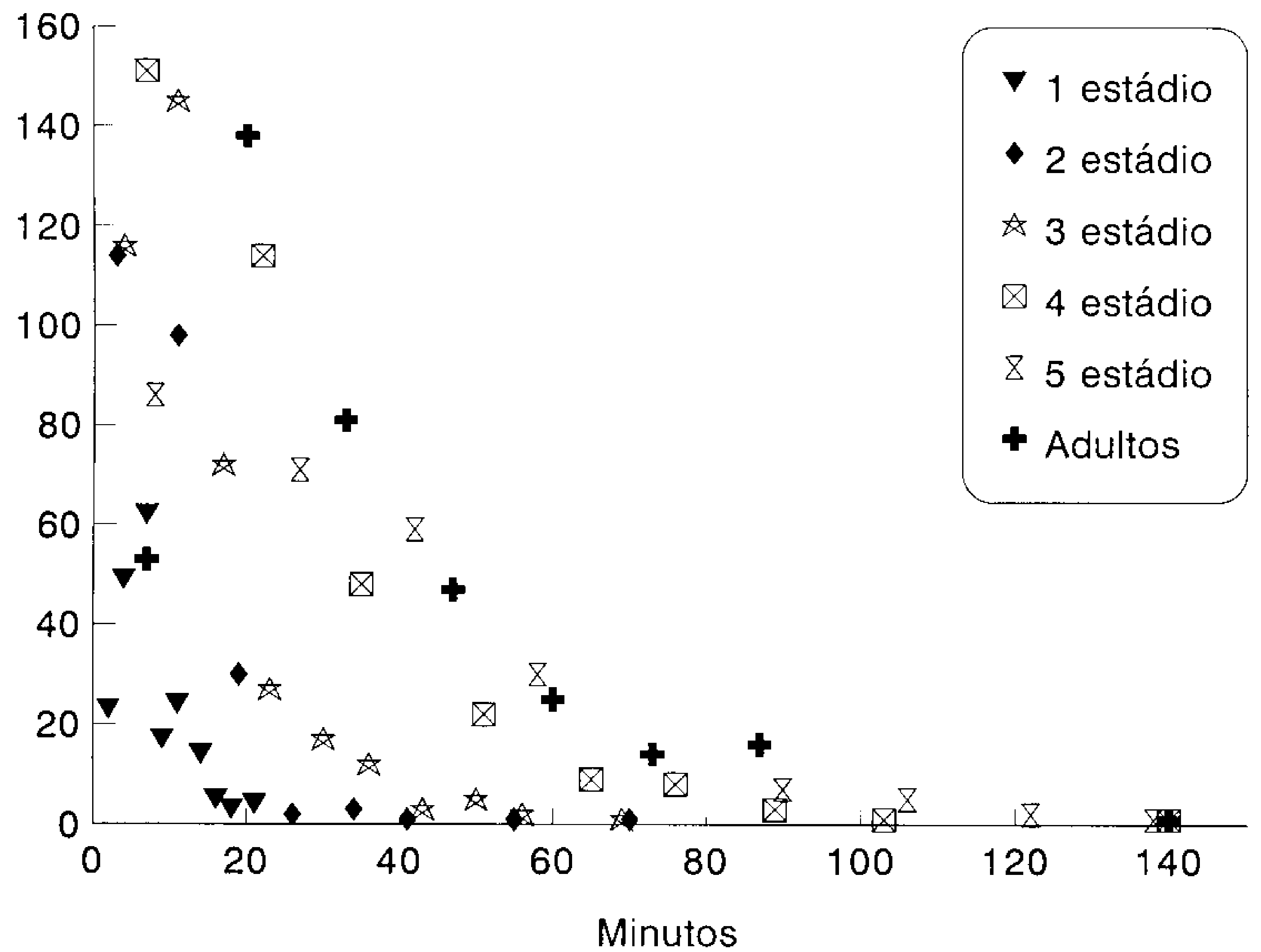

Fig. 2: duração do repasto em todas as fases do desenvolvimento de Triatoma nitida.

taria também a exposição dos insetos a riscos.

Embora algumas espécies possam atingir até o $4^{\circ}$ estádio com apenas um repasto por fase, há que se considerar a influência de fatores como temperatura e fonte alimentar, além do stress causado pela manipulação. Freitas et al. (1967) demonstraram que com alimentação semanal o ciclo de vida do $R$. neglectus Lent, 1954 foi mais curto quando comparado com a alimentação quinzenal. Mc Ewen e Lehane (1993) verificaram que ninfas de $5^{\circ}$ estádio

\section{TABELA II}

Número de repastos realizados em cada fase do desenvolvimento de Triatoma nitida

\begin{tabular}{lccccc}
\hline Fases & Min. & Máx. & $\overline{\mathrm{X}}$ & $\mathrm{S}$ & $\mathrm{N}$ \\
\hline $1^{\circ}$ estádio & 1 & 5 & 2,7 & 0,84 & 73 \\
$2^{\circ}$ estádio & 1 & 8 & 3,4 & 1,16 & 71 \\
$3^{\circ}$ estádio & 3 & 12 & 6,1 & 1,91 & 60 \\
$4^{\circ}$ estádio & 3 & 18 & 6,1 & 2,59 & 57 \\
$5^{\circ}$ estádio & 3 & 14 & 5,2 & 2,47 & 40 \\
Adultos & 1 & 26 & 10,1 & 6,65 & 37 \\
\hline
\end{tabular}

de $T$. infestans alimentadas com mais freqüência tinham seu desenvolvimento acelerado.

A quantidade e a fonte de sangue podem alterar, significativamente, além do tempo de desenvolvimento, a fecundidade e a capacidade de dispersão dos adultos pelo vôo (Ekkens 1981, Lehane e Schofield 1981,1982).

Costa et al. (1986, 1987), Diotaiuti e Dias (1987) e Costa e Jurberg (1990) demonstraram o melhor desenvolvimento de diversas espécies quando ali-

\section{TABELA III}

Duração das fases de desenvolvimento (em dias) de Triatoma nitida

\begin{tabular}{lccccc}
\hline Fases & Min. & Máx. & $\bar{X}$ & S & N \\
\hline $1^{0}$ estádio & 14 & 105 & 54,6 & 20,32 & 73 \\
$2^{\circ}$ estádio & 13 & 202 & 60,6 & 33,44 & 71 \\
$3^{\circ}$ estádio & 49 & 358 & 180,3 & 71,8 & 60 \\
$4^{0}$ estádio & 89 & 446 & 228 & 93,41 & 57 \\
$5^{\circ}$ estádio & 60 & 399 & 217,1 & 90,29 & 40 \\
Adultos & 26 & 457 & 193,2 & 115,15 & 39
\end{tabular}


mentadas em camundongos do que em pombos.

Duração do ciclo de vida - T. nitida apresentou um dos mais longos períodos de desenvolvimento já relatados na literatura. A média de duração do ciclo, da eclosão à fase adulta (até a data da morte) foi de 897,6 dias com um periodo mínimo de $523 \mathrm{e}$ máximo de 1394 dias. Estes resultados (Tabela III) aproximam-se dos obtidos por Dias (1955) e Souza et al. (1978) para T. sordida (Stal, 1859) e Heitzmann-Fontenelle (1983) para T. brasiliensis. Lent e Jurberg (1969) observando o ciclo do Panstrongylus geniculatus (Latreille, 1811), obtiveram resultados dilatados devido à necessidade de alta umidade para a criação dessa espécie.

A principal vantagem da criação individual é permitir a observação detalhada de inúmeros parâmetros biológicos importantes para a determinação do potencial vetorial da espécie. Entretanto, deve-se considerar a possibilidade de efeitos negativos, causados pelo manuseio excessivo e confinamento dos insetos.

Tolezano et al. (1984) atribuíram ao excesso de manuseio o aumento da mortalidade e a diminuição da fertilidade do $T$. infestans e Costa e Jurberg (1990) recomendaram cautela na aceitação dos resultados obtidos em criações individuais de C. lenti, devido ao stress causado pela manuseio, já que esta espécie se mostrou altamente sensível a qualquer estímulo externo que poderia, inclusive, provocar a morte dos insetos.

A alta mortalidade das ninfas de $5^{\circ}$ estádio, que foi da ordem de $30 \%$ (Fig. 4), foi também observada por outros autores. Barretto et al. (1981) destacaram o alto índice de mortalidade de Dipetalogaster maxima (Uhler, 1894) durante a muda, sobretudo no $5^{\circ}$ cstádio, mas não conseguiram explicação para tal fato. Costa et al. (1987) fizeram observações semelhantes sobre as anomalias ocorridas durante a muda e confirmaram a alta taxa de mortalidade do D. maxima nesta fase. Casos de ecdises defeituosas e interrupção do processo da muda imaginal, elevando a mortalidade, também foram registrados por Lent e Valderrama (1977) para três espécies do gênero Rhodnius e por Rocha et al. (1994) para $R$, pictipes. Rabinovich (1972) também fez referência à alta mortalidade do $T$. infestans no $5^{\circ}$ estádio, porém nada comentou a respeito de ecdises anormais.

Alguns autores atribuem às condições de criação apossimbióticas a alta mortalidade no $5^{\circ}$ estádio e na muda imaginal e atrasos no desenvolvimento (Brecher \& Wigglesworth 1944, Geigy et al. 1953, Harington 1960). Segundo Lake e Friend (1968), a ausência do microorganismo simbionte Nocardia rhodnii (Erikson. 1935) ocasionaria deficiência de vitaminas do complexo $\mathrm{B}$ em $R$. prolixus. Nyirady (1973) fez estudos comparativos com T. protracta, T. rubida (Uhler, 1894) e $R$.
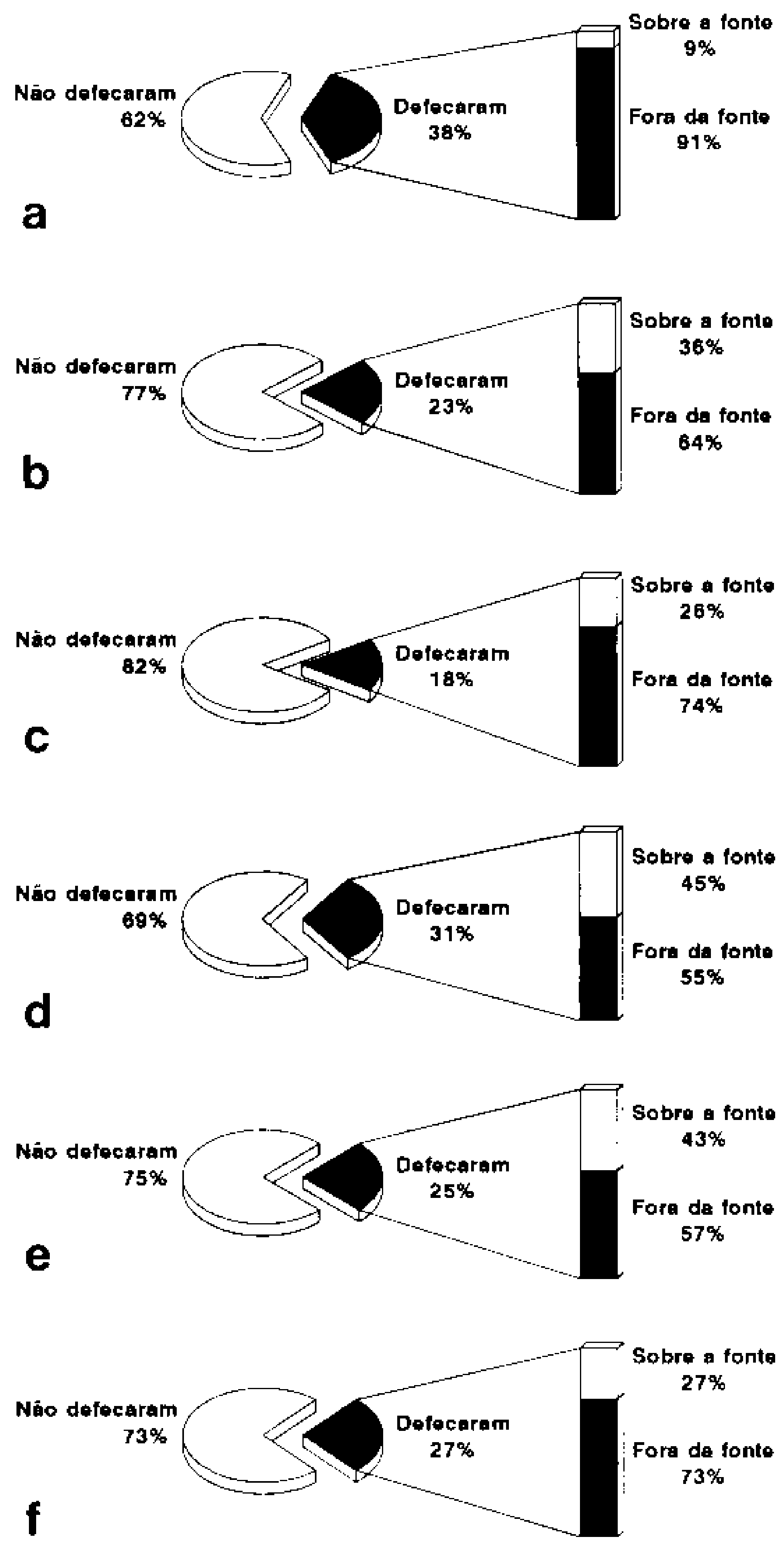

Fig. 3: percentual de defecações realizadas até $20 \mathrm{~min}$. após o repasto, e local do depósito das dejeçoes em cada fase do desenvolvimento de Triatoma nitida - a: $1^{\circ}$ estádio, b: $2^{\circ}$ estádio, c: $3^{\circ}$ estádio, d: $4^{\circ}$ estádio, e: $5^{\circ}$ estádio, $f:$ adultos.

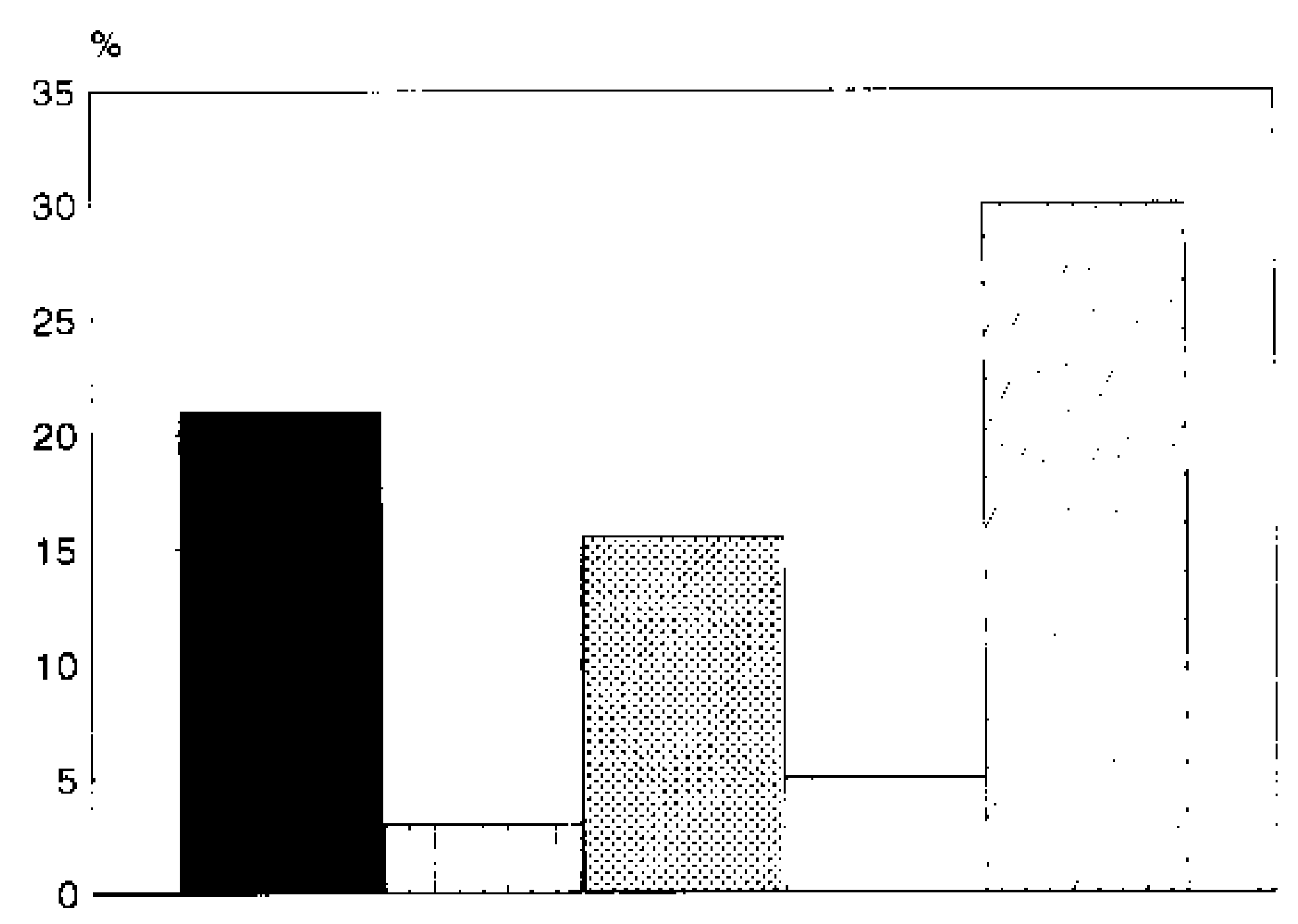

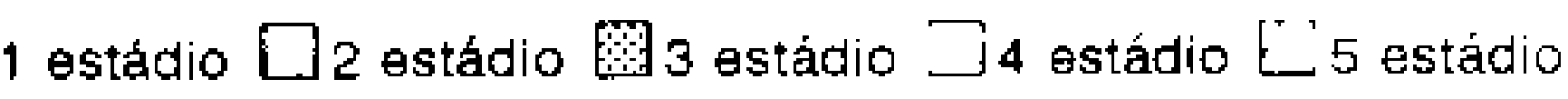

Fig. 4: percentual de mortalidade das ninfas de Triatoma nitida. 
prolixus criados de forma convencional $\mathfrak{e}$ assepticamente, com a finalidade de eliminar a $N$. rhodnii; acompanhando o desenvolvimento de quatro gerações de insetos em frascos estéreis e alimentados em camundongos germfree verificou que não houve alterações no desenvolvimento dos insetos.

No presente trabalho os insetos não foram criados em condições apossimbióticas, já que os ovos tiveram contato com as fezes e as ninfas provavelmente se contaminaram no momento da eclosão. Como não foi feito nenhum controle a respeito da contaminação por simbiontes, e diante da complexidade do tema, tornam-se necessários estudos adicionais, porém, acreditamos que o excessivo manuseio possa ter exercido alguma influência.

\section{AGRADECIMENTOS}

Ao Dr. Rodrigo Zeledón, da Universidade da Costa Rica pelos espécimes que originaram a colônia de $T$. nitida; ao técnico José Luis da Costa Giesteira, pelo auxílio no acompanhamento das observações e à estagiária Luciana da Fonseca Silva pelo auxílio na manutenção do insetário.

\section{RE FERÊNCIAS}

Barretto AC, Prata AR, Marsden PD, Cuba CC, Trigueira CP 1981. Aspectos biológicos e criação em massa de Dipetalogaster maximus (Uhler, 1894) (Triatominae). Rev Inst Med Trop São Paulo 23: 18-27.

Brasileiro VLF, Perondini ALP 1976. Biologia do Triatoma brasiliensis (Hemiptera, Reduviidae, Triatominae) I. Tempo de sucção e repleção de ninfas de $4^{\circ}$ e $5^{\circ}$ estádio. Rev bras Ent 18: 43-50.

Brecher G, Wigglesworth VB 1944. The transmission of Actinomyces rhodnii Erikson in Rhodnius prolixus Stal (Hemiptera) and its influence on the growth of the host. Parasitology 35: 220-224.

Carcavallo RU, Martinez AM 1972. Life cycles of some species of Triatoma (Hemiptera: Reduviidae). Can Entomol 104: 699-704.

Costa JM, Jurberg J 1990. Estudos bionômicos de Cavernicola lenti Barrett \& Arias, 1985 (Hemiptera, Reduviidae, Triatominae). Mem Inst Oswaldo Cruz 85: $357-366$

Costa JM, Jurberg J, Almeida JR 1986. Estudos bionômicos de Dipetalogaster maximus (Uhler, 1894) (Hemiptera-Triatominae). I - Influência da dieta sobre o ritmo de postura, viabilidade dos ovos, curva de fertilidade e mortalidade das fêmeas. Mem Inst Oswaldo Cruz 81: 365-380.

Costa JM, Jurberg J, Almeida JR 1987. Estudos bionômicos de Dipetalogaster maximus (Uhler, 1894) (Hemiptera-Triatominae). II - Influência da dieta sobre o ciclo biológico e resistência ao jejum. Mem Inst Oswaldo Cruz 82: 111-118.

Dias E 1952. Doença de Chagas nas Américas. III - Aménica Central. Rev Bras Malariol D trop 4: 75-84.

Dias E 1955. Notas sôbre o tempo de evolução de algu- mas espécies de Triatomíneos em laboratório. Rev Brasil Biol 15: 157-158.

Dias E 1956. Observações sôbre a eliminação de dejeções e tempo de sucção em alguns triatomineos sul-americanos. Mem Inst Oswaldo Cruz 54: 115 124

Dias E, Chandler AC 1949. Moléstias humanas transmitidas por hemípteros sugadores. Mem Inst Oswaldo Cruz 47: 403-422.

Diotaiuti L, Dias JCP 1987. Estudo comparativo do ciclo evolutivo de Rhodnius neglectus alimentados em pombos ou camundongos. Rev Soc Bras Med Trop 20: 95-100.

Ekkens DB 1981. Nocturnal fligths of Triatoma (Hemiptera: Reduviidae) in Sabino Canyon, Arizona. I Light collections. J Med Entomol 18: 21 1-227.

Freitas MG, Costa JO, Costa HMA 1967. Alguns aspectos da biologia do Rhodnius neglectus Lent, 1954 (Hemiptera, Triatominae) em condições de laboratório. I Evolução. Arq Esc Vet 19:81-87.

Geigy VR, Halff LA, Kocher V 1953. Untersuchungen uber die physiologischen Beziehungen awischen einen Ubertrager der Chagas - Krankheit Triatoma infestans und dessen Darmsymbionten. Schweizerische Med Wochenschrift 39: 928-930.

Gonzalez-Angulo W, Ryckman RE 1967. Epizootiology of Trypanosoma cruzi infections in Triatominae and man on the Yucatan Peninsula of Mexico. J Med Ent 4: 44-47.

Harington JS 1960. Studies on Rhodnius prolixus: growth and development of normal and sterile bugs, and the symbiotic relatronship. Parasitology 50: $279-286$

Heitzmann-Fontenelle TJ 1983/84. Bionomia comparativa de triatomíneos. II - Triatoma brasiliensis Neiva, 1911(Hemiptera, Reduviidae). Mem Inst Butantan 47/48: 157-164.

Juarez E 1970. Comportamento do Triatoma infestans sob várias condições de laboratório. Rev Saúde Públ $S$ Paulo 4: 147-166.

Jurberg J, Galvão C, Barth OM 1991. Estudo morfológico de ovos e ninfas de Triatoma nitida Usinger, 1939 (Hemiptera, Reduviidae,Triatominae). Rev Brasil Biol 51: 365-372.

Kirk ML, Schofield CJ 1987. Density-dependent timing of defaecation by Rhodnius prolixus, and its implications for the transmission of Trypanosoma cruzi. Trans R Soc Trop Med Hyg 81: 348-349.

Lafetá LP 1966. Sôbre a primeira alimentação da ninfa de $1^{\circ}$ estádio do Panstrongylus megistus Burmeister (1835). Rev Brasil Malariol D trop 18: 113-124.

Lake P, Friend WG 1968. The use of artificial diets to determine some of the effects of Nocardia rhodnii on the development of Rhodnius prolixus. J Insect Physiol 14: 543-562.

Lehane MJ, Schofield CJ 1981. Field experiments of dispersive flight by Triatoma infestans. Trans $R$ Soc trop Med Hyg 75: 399-400.

Lehane MJ, Schofield CJ 1982. Flight initiation in Triatoma infestans (KJug) (Hemiptera: Reduviidae). Bull Ent Res 72: 497-510.

Lent H, Jurberg J 1969. Observações sobre o ciclo 
evolutivo, em laboratório, do Panstrongylus geniculatus (Latreille, 1811) (Hemiptera, Reduviidae, Triatominae). An Acad Brasil Ciênc 41: 125-131.

Lent H, Jurberg J 1992. Estudo comparativo da genitália externa masculina deTriatoma neotomae Neiva, 1911 e Triatoma nitida Usinger, 1939 (Hemiptera, Reduviidae), Mem Inst Oswaldo Cruz 87: 123-130.

Lent H, Valderrama A 1977. Observações, em laboratório, sobre o ciclo evolutivo de Rhodnius prolixus Stal, $1859, R$ pictipes Stal, 1872 e $R$. neivai Lent, 1953. Rev Brasil Biol 37: 325-344.

Lent H, Wygodzinsky P 1979. Revision of the Triatominae (Hemiptera, Reduviidae), and their significance as vectors of Chagas' disease. Bull $\mathrm{Am}$ Mus Nat Hist 163: 123-520.

Leon JR 1959. Estado Actual de la Enfermedad de Chagas en Guatemala. Resumen Epidemiologico. Rev Goiana Med 5: 445-455.

I.eopoldo-e-Silva R, Corrêa FMA, Curi PP 1990. Innuência da precocidade do primeiro repasto no desenvolvimento pós-embrionário de Triatoma infestans Klug, 1834 e de Rhodnitus prolixus Stal, 1859 (Hemiptera, Reduviidade, Triatominae) em condições de laboratório. Rev bras Ent 34: 361-367.

McEwen PK, Lehane MJ 1993. The effect of varying feed interval on nymph development, subsequent adult flight behaviour and autogeny, in the triatominae bug Triatoma infestans (Klug) (Hem., Reduviidae). J Appl Ent 115: 90-96.

Nyirady SA 1973. The germfree culture of three species of Triatominae: Triatoma protracta (Uhler); Triatoma rubida (Uhler) and Rhodnius prolixus Stal. J Med Ent 10: 417-448.

Ozete HJ 1981. Observaciones sobre la biologia de Triatoma flavida Neiva, 1911 en Cuba. Rev Cub Med Trop 33: 42-50.

Perlowagora-Szumlewicz A 1969. Estudos sobre a biologia do $T$. infestans, o principal vetor da doença de Chagas no Brasil (Importância de algumas de suas características biológicas no planejamento de es- quemas de combate a esse vetor). Rev Brasil Malariol D trop 21: 117-159.

Pippin WF 1970. The biology and vector capability of Triatoma sanguisuga texana Usinger and Triatoma gerstaeckeri (Stal) compared with Rhodnius prolixus (Stal) (Hemiptera: Triatominae). JMed Ent 7: $30-45$

Rabinovich JE 1972. Vital statistics of Triatominae (Hemiptera: Reduviidae) under laboratory conditions I Triatoma infestans Klug. J Med Ent 9: $351-370$.

Rocha DS, Galvão C, Jurberg J 1994. Biologia do Rhodnius pictipes Stal, 1872 em condições de laboratório (Hemiptera, Reduviidae, Triatominae). Mem Inst Oswaldo Cruz 89: 265-270.

Silva HHG, Silva IG 1991. Ciclo evolutivo de Triatoma dimidiata capitata Usinger, 1944 (Hemiptera, Reduviidae). An Soc Ent Brasil 20: 405-410.

Souza JMP, Rodrigues VLCC, Rocha-e-Silva EO 1978. Triatoma sordida - considerações sobre o tempo de vida das formas adultas e sobre a oviposição das fềmeas. Rev Saúde Públ São Paulo 12: 291-296.

Tolezano JE, Araújo MFS, Chieffi PP, Valentim AM, Ribeiro SS 1984. Variáveis relacionadas ao desenvolvimento de Triatoma infestans Klug, $1834 \mathrm{em}$ condições de laboratório. Efeitos da manipulação na sobrevivência e na fertilidade. Rev Inst Adolfo Lutz 44: 81-86.

Wood SF 1951. Importance of feeding and defecation times of insect vectors in transmission of Chagas' disease. $J$ econ Ent 44: 52-54.

Wood SF 1976. Body weight and blood meal size in Triatoma protracta (Hemiptera: Reduviidae). Ann Entomol Soc Am 69: 632-634.

Zárate LG, López GM, Ozuna MC, Santiago GG, Zárate RJ 1984. The biology behaviour of Triatoma barberi (Hemiptera: Reduviidae) in Mexico. IV. Feeding and defecation patterns. J Med Entomol 21: 548-560.

Zeledón R, Alvarado R, Jirón LF 1977. Observations on the feeding and defecation patterns of three triatominae species (Hemiptera:Reduviidae). Acta Tropica 34: $65-77$ 